\title{
Basic Theorems on the Backoff Process in 802.11*
}

\author{
Jeong-woo Cho and Yuming Jiang \\ Centre for Quantifiable Quality of Service in Communication Systems \\ Norwegian University of Science and Technology (NTNU), Norway
}

\section{INTRODUCTION}

Since its introduction, the performance of IEEE 802.11 has attracted a lot of research attention and the center of the attention has been the throughput. For throughput analysis, in the seminal paper by Kumar et al. 8], they axiomized several remarkable observations based on a fixed point equation (FPE). Above all, one of the key findings of [8] is that the full interference model, also called the single-cell model [8], in 802.11 networks leads to the backoff synchrony property which implies the backoff process can be completely separated and analyzed through the FPE technique. To date, however, only the uniqueness of the fixed point has been proven to hold under some mild assumptions [8, Theorem 5.1], and we still need an answer to the following fundamental question:

Q1: "Exactly under which conditions the fixed point equation technique is valid?" (to be answered in Theorem 1)

An intriguing notion, called short-term fairness, has been introduced in some recent works [2,4]. It can be easily seen that this notion pertains to a purely backoff-related argument also owing to the backoff synchrony property in the full interference model [8]. The two papers [2, 4] considered the same situation where only two wireless nodes contend for the medium. The former [2] claimed and conjectured through simulations that the summation of the backoff values generated per a packet, denoted by $\Omega$, is uniformly distributed because the initial backoff is uniformly distributed while the latter 4] conjectured based on their experiments that $\Omega$ is exponentially distributed in the sense that its coefficient of variation $(\mathrm{CV})$ is one. This left room for misunderstandings about the backoff distribution:

Q2: "One of the two works is incorrect?" (to be answered in Theorem 21)

In addition, the two works [2] defined $\mathrm{P}[z \mid \zeta]$ as the probability that other nodes transmits $z$ packets while a tagged node is transmitting $\zeta$ packets and acquired the expression of $\mathrm{P}[z \mid \zeta]$ valid only for the two node case. It is natural to ask the following pertinent questions:

Q3: "Can we develop an analytical model for short-term fairness?" (to be answered in Theorem 3)

Q4: "When does the short-term fairness undergo a dramatic

\footnotetext{
*A part of this work was done when the first author was with the School of Computer and Communication Sciences, Ecole Polytechnique Fédérale de Lausanne (EPFL), Switzerland.

$\dagger$ "Centre for Quantifiable Quality of Service in Communication Systems, Centre of Excellence" appointed by The Research Council of Norway, and funded by The Research Council, NTNU and UNINETT. http://www.q2s.ntnu.no
}

change?" (to be answered in Theorem 5)

One interesting finding in 9] is that the access delay has a wide-sense heavy-tailed distribution 9, Theorem 1] which means that its moment generating function diverges (to infinity), i.e., $\int_{0}^{\infty} \mathrm{e}^{t x} f(x) d x=\infty, \forall t>0$, where $f(x)$ is the corresponding pdf (probability density function). One should be careful in interpreting this finding because the wide-sense heavy-tailedness does not imply strict sense heavytailedness, which roughly means the ccdf (complementary cumulative distribution function) is of Pareto-type [6] with an exponent over $(-2,0)$. Consequently, the discussion poses the following challenge which is undoubtedly a tantalizing question.

Q5: "What is the distribution type of the delay-related variables?" (to be answered in Theorem 4)

This paper discovers fundamental principles of the backoff process and provides answers to the open questions highlighted above. These results not only help us to get the complex details of the backoff process in 802.11 into perspective under one framework, but also remove possible misunderstandings caused by the two recent works [2, 4. Proofs of theorems and ns-2 simulation results are omitted; we refer the reader to our technical report [5].

\section{MEAN FIELD TECHNIQUE REVISITED}

Though a de facto standard now, we revisit mean filed technique here since it supports our theorems in later sections. Let $X_{n}(t)$ denote the backoff stage of node $n$ at continuous time $t$. We assume that there are $K+1$ backoff stages, i.e., $X_{n}(t) \in\{0,1, \cdots, K\}$. The occupancy measure (or empirical measure) of backoff stage $k$ at $t$ is defined as $\phi_{k}(t):=\frac{1}{N} \sum_{n=1}^{N} 1_{\left\{X_{n}(t)=k\right\}}$ where $1_{\{\cdot\}}$ is the indicator function. The beauty of the intensity scaling adopted in [1] lies in that (i) the mean field limit of the occupancy measure, which we also denote by $\phi_{k}(t)$ at the risk of notational abuse, evolves according to the following set of mean field ordinary differential equations (MFODE) and (ii) the distribution of $X_{n}(t)$ of every node $n$ follows the distribution of $\phi(t):=\left(\phi_{0}(t), \cdots, \phi_{K}(t)\right):$

$$
\begin{aligned}
\frac{d \phi_{k}}{d t}(t) & =p_{k-1} \phi_{k-1}(t) \gamma(t)-p_{k} \phi_{k}(t), \\
\frac{d \phi_{0}}{d t}(t) & =\bar{p}(t)(1-\gamma(t))-p_{0} \phi_{0}(t)+p_{K} \phi_{K}(t) \gamma(t),
\end{aligned}
$$

where (1) holds for $k \in\{1, \cdots, K\} \cdot \bar{p}(t)$ and $\gamma(t)$ respectively designate the average attempt rate and collision probability of every node. Though we are usually more interested in decoupling approximation in which we need to consider only the stationary points, Benaïm and Le Boudec [1] 
stressed that the stability of mean field stationary points for finite $K$ which we prove in the following is required for decoupling approximation.

\section{Theorem 1 (Global Stability of MFODE)}

If $\left\{p_{k}\right\}, 0 \leq k \leq K$, is a nonincreasing sequence, the mean field independence in the stationary regime holds for $K>0$.

Remark 1 R:1.1 [Answer to Q1] The beauty of Theorem 1 is that it supports us to apply decoupling approximation in the rest of the analysis, which is that the backoff process at one node can be treated as if it is independent from the backoff processes at other nodes when $N$ is large.

\section{BACKOFF ANALYSIS}

We assume that the backoff value generated at backoff stage $k$ has a pdf (probability density function) $f_{k}(\cdot)$ with mean $1 / p_{k}$ and variance $v^{2} / p_{k}^{2}$. Note that $v$ corresponds to the CV (coefficient of variation).

Let $\Omega$ and $f_{\Omega}(\cdot)$ respectively denote the sum of the backoff values generated for a packet, and its pdf. Also denote by $\bar{\Omega}$ its mean and $\sigma_{\Omega}^{2}$ its variance. It is our pleasure to prove in the following that both of the two works [2,4] are correct. Theorem 2 (Per-Packet Backoff Principle)

The per-packet backoff of every node, $\Omega$, has the pdf

$$
f_{\Omega}(b)=f^{* K}(b) \gamma^{K}+(1-\gamma) \sum_{k=0}^{K-1} f^{* k}(b) \gamma^{k}
$$

where $f^{* k}(b):=\left(f_{0} * \cdots * f_{k}\right)(b)$ is the convolution of $k+1$ functions. Moreover, its mean and variance are given by

$$
\begin{aligned}
\bar{\Omega} & =\sum_{k=0}^{K} \frac{\gamma^{k}}{p_{k}} \\
\sigma_{\Omega}^{2} & =\left(1+v^{2}\right)\left(\sum_{k=0}^{K} \frac{\gamma^{k}}{p_{k}^{2}}\right)+2\left(\sum_{k=1}^{K} \frac{\gamma^{k}}{p_{k}} \sum_{i=0}^{k-1} \frac{1}{p_{i}}\right)-(\bar{\Omega})^{2} .
\end{aligned}
$$

Remark 2 R2.1 [Answer to Q2] Consider the case $N=2$. It can be computed from (3) and (4) that $\Omega$ is approximately uniformly in $802.11 \mathrm{~b}$, and exponentially distributed in $802.11 \mathrm{a} / \mathrm{g}$ in the sense that $v_{\Omega}:=\sigma_{\Omega} / \bar{\Omega} \approx 0.7$ (though slightly larger than $1 / \sqrt{3}$ ) and $v_{\Omega} \approx 1.0$, respectively, due to different initial contention windows $\left(2 / p_{0}=32\right.$ and 16$)$.

Denoting by $n \in\{1, \cdots, N-1\}$ the indices of other nodes, WLOG, we consider a tagged node whose index is $N$. For short-term fairness analysis, we consider the number of packets transmitted by $N-1$ other nodes while the tagged node is transmitting $\zeta$ packets. Define $Z_{n}$ as the number of intertransmissions of nodes $n \in\{1, \cdots, N-1\}$ and let

$$
Z=\sum_{n=1}^{N-1} Z_{n}, \quad \mathrm{P}_{N}[z \mid \zeta]:=\mathrm{P}\left[\sum_{n=1}^{N-1} Z_{n}=z \mid Z_{N}=\zeta\right]
$$

which is called inter-transmission probability, the probability that $N-1$ nodes transmits $z$ packets given the tagged node $N$ transmits $\zeta$ packets at the risk of notational abuse.

Deriving an exact closed-form solution of $\mathrm{P}_{N}[z \mid \zeta]$ for an arbitrary number of nodes $N$ necessitates the computation of the convolutions of uniform pdfs with different means given by (2). It is known that this computation yields more complicated expressions than expected. We suggest a theoretical alternative - the backoff procedure of other nodes can be regarded as a superposition of $N-1$ statistically identical point processes, i.e.,

$$
A(t):=\sum_{n=1}^{N-1} A^{n}(t)
$$

where the backoff procedure of node $n$ is viewed as a stationary simple point process $A^{n}(t)$ that counts the number of arrivals during the interval $(0, t]$. The key observation is that the entropy of the superposition point process $A(t)$ increases with $N-1$, based on which the following result is derived, which is the principle of the backoff process about the intertransmission probability and characterizes the short-term fairness performance of 802.11.

\section{Theorem 3 (Inter-Transmission Principle)}

Suppose $\zeta \gg 1$ and $N \gg 1$. The inter-transmission probability can be approximated as follows.

$$
\begin{aligned}
& \mathrm{P}_{N}[z \mid \zeta] \approx \\
& \quad \int_{-\infty}^{\infty} \mathbf{N m}(x) \cdot \mathbf{P s}\left((N-1)\left(\zeta+\sqrt{\zeta} v_{\Omega} x\right), z\right) d x
\end{aligned}
$$

where $\mathbf{N m}(x):=\frac{1}{\sqrt{2 \pi}} \mathrm{e}^{-\frac{x^{2}}{2}}$ and $\operatorname{Ps}(\lambda, z):=\frac{1}{z !} \mathrm{e}^{-\lambda} \lambda^{z}$. Moreover, the mean and variance are given by

$$
\bar{Z} \approx(N-1) \zeta, \sigma_{Z}^{2} \approx(N-1) \zeta+(N-1)^{2} \zeta \cdot v_{\Omega}^{2} .
$$

Remark 3 R 3,1 [Answer to Q3] For the case when the intensity of a Poisson point process is a random measure on the time space, the process is called a Cox process or doubly stochastic Poisson process. It is interesting to note that the inter-transmission process in 802.11 can be viewed as if it is a Cox process on the line $(0, \zeta]$ with an average random intensity $\bar{\lambda}(\zeta)$, that is defined as the number of transmissions of the aggregate per a transmission of the tagged node and given by:

$$
\bar{\lambda}(\zeta) \stackrel{\mathrm{d}}{=} \mathbf{N}\left(N-1,(N-1)^{2} v_{\Omega}^{2} / \zeta\right),
$$

Here $\mathbf{N}\left(\mu, \sigma^{2}\right)$ denotes the Gaussian random variable with mean $\mu$ and variance $\sigma^{2}$.

R[3. 2 The CV of $Z$ can be approximated by

$$
v_{Z}:=\frac{\sigma_{Z}}{Z} \approx \sqrt{\frac{1}{(N-1) \zeta}+\frac{v_{\Omega}^{2}}{\zeta}} \approx v_{\Omega} / \sqrt{\zeta} .
$$

for large $N$. This particularly reveals that the dominating factor in deciding the short-term fairness in 802.11 is the $\mathrm{CV}$ of the per-packet backoff $\left(v_{\Omega}\right)$ and the number of transmissions of the tagged node $(\zeta)$. Consequently, $Z$ is approximately Gaussian for large $N$ :

$$
Z \stackrel{\mathrm{d}}{\approx} \mathbf{N}\left((N-1) \zeta,(N-1)^{2} \zeta v_{\Omega}^{2}\right)
$$

That is, (6) manifests that the Poissonian benefit, led to by the increased entropy of the aggregated processes, is counterbalanced by, and after all dominated by the detrimental effect of the collisions between nodes.

\section{ASYMPTOTIC ANALYSIS $(K=\infty)$}

Although the power-law like behavior of access delays was identified only through simulations in a few works 9, to the best of our knowledge, none of them proved that the delay or backoff duration has a power-law tail. We prove that the per-packet backoff distribution has a power tail by lighting upon the fact that the moment generating function has a recursive relation, and by applying the theory of regular variation [3] and the less-known modified Tauberian theorem of Bingham \& Doney [5]. It is important to note that this theorem is a prerequisite for mathematical analysis of Noah effect, which implies strict-sense heavy-tailedness. 
Theorem 4 (Power Tail Principle)

Suppose $K=\infty$ and define $\alpha:=-(\log \gamma) / \log m$. Note that $\alpha>1$ by [5, Lemma 4]. Then, the per-packet backoff $\Omega$ has a Pareto-type tail with an exponent of $-\alpha$. Formally,

$$
F_{\Omega}^{c}(x):=\int_{x}^{\infty} f_{\Omega}(x) d x \sim x^{-\alpha} \ell(x) .
$$

The notation $f(x) \sim g(x)$ means $\lim _{x \rightarrow \infty} f(x) / g(x)=1$, and $\ell(x)$ is slowly varying [3].

Remark 4 R4.1 This principle, formulated in terms of the ccdf (complementary cumulative distribution function) $F_{\Omega}^{c}(\cdot)$, not only defines a fundamental characteristic of delay but also lays the groundwork for further analysis using regular variation theory.

R4,2 [Answer to Q5] This clear-cut result reveals the statistical attribute of $\Omega$ : it has a Pareto-type distribution whose main parameter is $-\alpha$. As compared with the preliminary result derived in 9 for wide-sense heavy-tailedness corresponding to only part of [5, Theorem 4], Theorem 4 extends to much larger extent by proving the strict-sense heavy-tailedness of $\Omega$ for $\alpha \in(1,2)$.

Let $\mathbf{S}_{\alpha}(\sigma, \beta, \mu)$ denote Lévy $\alpha$-stable laws whose four parameters are: the index $\alpha$; the scale parameter $\sigma$; the skewness parameter $\beta$; and the mean $\mu$. If $X_{1}, \cdots, X_{n}$ are i.i.d. copies of $\mathbf{S}_{\alpha}(\sigma, \beta, \mu)$, they satisfy the stability property which takes the following form:

$$
\sum_{i=1}^{m}\left(X_{i}-\mu\right) \stackrel{\mathrm{d}}{=} m^{\frac{1}{\alpha}}\left(X_{1}-\mu\right) .
$$

Since the discovery of the self-similarity in the Internet, the probabilistic community has been concerned with the limit processes of aggregate renewal processes under different limit regimes. Recently, Kaj [7] has established a fundamental connection [7. Theorem 1]; it turns out that for $\alpha \in(1,2)$, the superposition process $A(t)$ defined in (5) exhibits long-range dependence due to the heavy power tail of inter-arrival times $\Omega$, meaning that, for large $N$ and $\zeta$,

$$
A(\zeta \tau) \stackrel{\mathrm{d}}{\approx}(N-1) \zeta \tau-\zeta c \mathbf{Y}_{\alpha}(\tau / c)
$$

where $\tau:=t / \bar{\Omega}$ is scaled time-slots, $\mathbf{Y}_{\alpha}(\cdot)$ belongs to the family of Intermediate Telecom process of index $\alpha$ whose cgf (cumulant generating function) takes the following form

$$
\begin{aligned}
& \log \mathrm{E}\left[\mathrm{e}^{\theta \mathbf{Y}_{\alpha}(\tau)}\right]=\frac{\tau^{1-\alpha}}{\alpha-1}\left(\mathrm{e}^{\theta \tau}-1-\theta \tau\right) \\
& +\int_{0}^{\tau}\left(\mathrm{e}^{\theta x}-1-\theta x\right)\left(\alpha \tau x^{-\alpha-1}+(2-\alpha) x^{-\alpha}\right) d x,
\end{aligned}
$$

and $c=\left\{(N-1) \bar{\Omega}^{-\alpha} \ell(\zeta \bar{\Omega})\right\}^{1 /(\alpha-1)} / \zeta$. Combining the theories introduced so far, we have the last principle.

Theorem 5 (Asym. Inter-Transmission Principle)

Suppose $K=\infty, \zeta \gg 1$ and $N \gg 1$. The inter-transmission probability can be approximated as follows. If $\alpha \in(2, \infty)$, Theorem 3 holds again. However if $\alpha \in(1,2)$,

$$
\mathrm{P}_{N}[z \mid \zeta] \approx \int_{-\infty}^{\infty}\left\{\int_{q_{-}(y)}^{q_{+}(y)} \mathbf{T c}^{\tau(y) / c}(x) d x\right\} \cdot \mathbf{L v}(y) d y
$$

where

$$
\begin{aligned}
q_{ \pm}(y) & :=-\{z \mp \delta-(N-1) \zeta \cdot \tau(y)\} /(\zeta c), \\
\tau(y) & :=1+\zeta^{(1-\alpha) / \alpha} \ell_{0}(\zeta) \cdot y
\end{aligned}
$$

$\ell_{0}(\cdot)$ is slowly varying at infinity, $\mathbf{T} \mathbf{c}^{\tau}(\cdot)$ is the pdf of $\mathbf{Y}_{\alpha}(\tau)$, and $\mathbf{L v}(\cdot)$ is the pdf of $\mathbf{S}_{\alpha}(1,1,0)$ whose index is $\alpha=-\frac{\log \gamma}{\log m}$. Remark 5 R5, 1 Similar to Remark 3, it can be verified that $Z$ becomes approximately Lévian for very large $\zeta$ (See [5] for details.):

$$
Z \stackrel{\mathrm{d}}{\approx} \mathbf{S}_{\alpha}\left((N-1) \zeta^{\frac{1}{\alpha}} \ell_{0}(\zeta), 1,(N-1) \zeta\right)
$$

This manifests the heavy-tail of $Z$, i.e., as $x \rightarrow \infty$,

$$
\mathrm{P}[Z>x] \approx \zeta\left\{(N-1) \ell_{0}(\zeta)\right\}^{\alpha} C_{\alpha} \cdot x^{-\alpha}
$$

where $C_{\alpha}=\frac{\alpha-1}{\Gamma(2-\alpha) \sin (\pi(\alpha-1) / 2)}$.

R5.2 The representation (11) reveals the striking similarity between the ccdfs of $\Omega$ and $Z$. That is, if $\alpha \in(1,2)$, in terms of regular variation theory, both are regularly varying of index $-\alpha$, and in Mandelbrot's terminology, Noah effect of $\Omega$ infiltrates into $Z$.

R[5.3 [Answer to Q4] In brief, the inter-transmission probability bifurcates into two different categories at $\alpha=2$ (or $\left.\gamma=1 / \mathrm{m}^{2}\right)$. Plainly speaking, if $\gamma<1 / \mathrm{m}^{2}, Z$ can still be approximated by the Gaussian distribution in (7), otherwise 802.11 suffers from extreme unfairness containing precursors of power-tailed characteristics such as infinite variances and the skewness $(\beta=1)$.

R[5.4 The skewness induces leaning tendency and directional unfairness. The leaning tendency implies the distribution is heavily leaning to the left, and the tendency increases as $\alpha$ decreases. The directional unfairness implies that while the right part of the inter-transmission probability $z \in(\bar{Z}, \infty)$ has a heavy power tail given by (11), its left part $z \in(-\infty, \bar{Z})$ decays faster than exponentially.

R5.5 Finally, (9) provides concrete evidence of the longrange dependence inherent in 802.11. Remarkably, though the process $\mathbf{Y}_{\alpha}(\cdot)$ exhibits long-range dependence in the sense that it has the same covariance as a multiple of fractional Brownian motion of index $H=(3-\alpha) / 2$, it is not self-similar.

\section{REFERENCES}

1] M. Benaim and J.-Y. Le Boudec. A class of mean field interaction models for computer and communication systems. Perf. Eval., 65(11-12):823-838, Nov. 2008.

[2] G. Berger-Sabbatel, A. Duda, O. Gaudoin, M. Heusse, and F. Rousseau. Fairness and its impact on delay in 802.11 networks. In Proc. IEEE Globecom, Nov. 2004.

[3] N. Bingham, C. Goldie, and J. Teugels. Regular Variation. Cambridge University Press, 1989.

[4] M. Bredel and M. Fidler. Understanding fairness and its impact on quality of service in IEEE 802.11. In Proc. IEEE Infocom, Apr. 2009.

[5] J. Cho and Y. Jiang. Fundamentals of the backoff process in 802.11. available at http://arxiv.org/abs/0904.4155 May 2009.

[6] M. Crovella and A. Bestavros. Self-similarity in world wide web traffic: Evidence and possible causes. ACM/IEEE Trans. Networking, 5(6):835-846, Dec. 1997.

[7] I. Kaj. Limiting fractal random processes in heavy-tailed systems. Fractals in Engineering, Springer-Verlag London, pp. 199-218, 2005.

[8] A. Kumar, E. Altman, D. Miorandi, and M. Goyal. New insights from a fixed-point analysis of single cell IEEE 802.11 WLANs. ACM/IEEE Trans. Networking, 15(3):588-601, June 2007.

[9] T. Sakurai and H. Vu. MAC access delay of IEEE 802.11 DCF. IEEE Trans. Wireless Commun., 6(5):1702-1710, May 2007. 